\title{
Exotic and Conventional Bottomonium Physics Prospects at Belle II
}

\author{
Todd K. Pedlar ${ }^{1, *}$ \\ ${ }^{1}$ Luther College Department of Physics \\ 700 College Drive \\ Decorah, Iowa 52101 USA
}

\begin{abstract}
The Belle II experiment, being constructed at the KEK laboratory in Japan, represents a substantial upgrade of both the Belle detector and the KEKB accelerator. It is expected that Belle II will collect 50 times more data than existing B-Factory samples beginning in 2019. Belle II is uniquely capable of studying the so-called "XYZ" particles: heavy exotic hadrons consisting of more than three quarks. First discovered by Belle, these now number in the dozens, and represent the emergence of a new category within Quantum Chromodynamics. In this article we summarize the capabilities of Belle II to explore both exotic and conventional bottomonium physics, with a particular focus on the physics reach of the first data, where opportunities exist to make an immediate impact in this area.
\end{abstract}

\section{Introduction}

While the Belle experiment's principal focus has been on the observation of $\mathrm{CP}$ violation and rare decays of $\mathrm{B}$ mesons, it has since its inception been the source of a significant number of important observations in both conventional and exotic quarkonia. These results include several first observations, and a number of measurements which are the most precise to date of several conventional bottomonium resonance parameters and branching fractions. The results of our study have also left a number of open questions which Belle II uniquely can answer. Here at the end of 2017, we are now are rapidly approaching the beginning of the Belle II experiment. Currently, the experiment is between two periods of phased commissioning of both detector and accelerator, the second of which is set to begin in early 2018, with full physics data taking with the completed Belle II detector planned for early 2019.

In what follows, we will introduce briefly the changes made between Belle I and Belle II, and upgrades to the KEKB accelerator which has now become SuperKEKB. We then discuss a number of observations made by the Belle Collaboration, and use them as a foundation from which to point out the prospects for new studies of both conventional and exotic bottomonia which are planned for Belle II. 

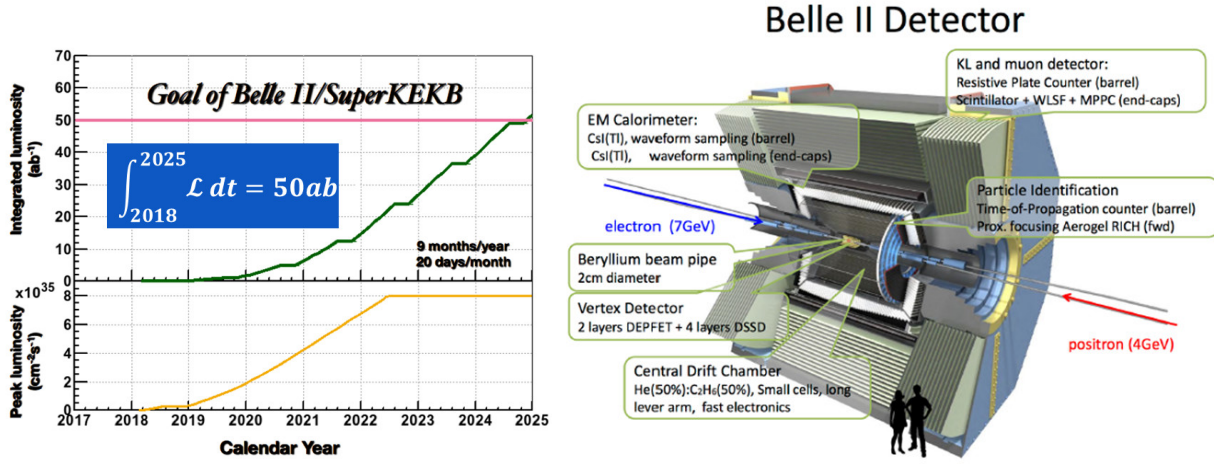

Figure 1. (left) Current luminosity estimates for Belle II. (right) Schematic of the Belle II detector.

\section{The Belle II Experiment}

\subsection{The SuperKEKB Accelerator}

The KEKB accelerator, which provided $e^{+} e^{-}$collisions for the Belle Experiment, produced the highest instantaneous luminosity seen in any experiment, reaching a peak value of $2.11 \times$ $10^{34} \mathrm{~cm}^{-2} \mathrm{~s}^{-1}$, and enabling Belle to integrate nearly $1 \mathrm{ab}^{-1}$ over its running period, mostly collecting data at $\Upsilon(4 \mathrm{~S})$, but also collecting the world's largest samples to date at the $\Upsilon(1 \mathrm{~S})$ and $\Upsilon(2 S)$ resonances, and in the $\Upsilon(5 S)$ and $\Upsilon(6 S)$ regions.

With upgrades to the accelerator[1], chiefly those which enable SuperKEKB to utilize the nanobeam scheme pioneered by P. Raimondi[2], it is expected that instantaneous luminosities for Belle II will be larger by approximately a factor of 40. Over the course of the running period planned for Belle II, a target of approximately $50 \mathrm{ab}^{-1}$ in total is expected to be integrated (See Fig. 1).

\subsection{The Belle II Detector}

In order to take advantage of the much increased luminosity afforded by SuperKEKB, we have upgraded the entire Belle detector system. The new detector sits inside the same superconducting solenoid magnet with the iron return yoke, and all components have either been replaced with different detectors or considerably upgraded. The complete Belle II detector system is discussed in full in the Belle II Technical Design Report [3].

One of the most significant impacts of the greater instantaneous luminosity Belle II will see is substantially increased background levels, which will lead to an increase in occupancy and radiation damage, fake hits and pile-up noise in the electromagnetic calorimeter, and neutron induced hits in the muon detection system. New readout electronics for most of the detector and a new trigger system have been introduced in order to compensate for the higher backgrounds. In addition, much improved hadron identification systems have been built, and, as the previous Belle detector, cover nearly the full $4 \pi$ steradians.

\subsection{Early Belle II Run Plan}

The commissioning phases of Belle II prior to commencement of physics data runs are denoted Phase I and Phase II. Phase I concluded in 2017, and involved only the commissioning

\footnotetext{
*e-mail: pedlto01@luther.edu
} 
Table 1. Summary of data samples collected by CLEO, Belle, and BaBar, together with potential samples collected by Belle II. The numbers reported are luminosities in $\mathrm{fb}^{-1}$, and the number of decays (in millions) of the produced bottomonium state is given in the parentheses adjacent to the reported luminosity.

\begin{tabular}{lllllll}
\hline Expt & $\Upsilon(1 \mathrm{~S})$ & $\Upsilon(2 \mathrm{~S})$ & $\Upsilon(3 \mathrm{~S})$ & $\Upsilon(4 \mathrm{~S})$ & $\Upsilon(5 \mathrm{~S})$ & $\Upsilon(6 \mathrm{~S})$ \\
\hline CLEO & $1.2(21)$ & $1.2(10)$ & $1.2(5)$ & $16(17.1)$ & $0.1(0.4)$ & - \\
BaBar & - & $14(99)$ & $30(122)$ & $433(471)$ & $R_{b}$ scan & $R_{b}$ scan \\
Belle & $6(102)$ & $25(158)$ & $3(12)$ & $771(772)$ & $121(36)$ & 5.5 \\
\hline Belle II & - & - & $300(1200)$ & $50000(54000)$ & $2000(600)$ & $100+400($ scan $)$ \\
\hline
\end{tabular}

of SuperKEKB running in single-beam mode, and a specially-built commissioning detector known as BEAST II [4]. Phase II running is expected to commence in January, 2018, and run through summer 2018, and will involve the commissioning of the fully instrumented complement of outer detectors and a partially instrumented inner detector system. During Phase II, the SuperKEKB final focusing magnets will be commissioned and the accelerator will move from single-beam to colliding beam operations for the first time. In this phase, a special configuration of the Beast II commissioning detectors, which will include partial instrumentation of the vertex region by pixel and silicon vertex components like those in the final Belle II vertex system, will be used to study beam-related backgrounds in colliding beam operations.

It is expected that despite the fact that beam collisions will not be optimal in Phase II, and the full inner detectors are not present, Belle II may be able to produce some data useful for physics analyses. Physics running in earnest in Phase III, however, will not begin until after the inner detector region is fully instrumented following the summer shutdown in 2018, and is expected to begin in early 2019. It is during the early running periods of Belle II, when the instantaneous luminosity of SuperKEKB has yet to reach the design specifications, that uniquely large samples of $\Upsilon(3 S), \Upsilon(6 S)$, and large fine scans in the $\Upsilon(6 S)$ region may be accumulated and useful for bottomonium studies - the primary $\Upsilon(4 S)$ data sample and a large $\Upsilon(5 S)$ sample that may be collected later will also make possible some of the studies discussed below. A table illustrating potential Belle II data samples is presented as Table 1.

\section{Studies of the Exotic $Z_{b}(10610)$ and $Z_{b}(10650)$ States}

Among the most exciting observations we have made at Belle in bottomonium spectroscopy, in addition to our discovery of the singlet-P $h_{b}(\mathrm{nP})$ states[5] in $\Upsilon(5 \mathrm{~S})$ dipion transitions (See Fig. 2), were the manifestly exotic $Z_{b}$ states lying above open-bottom threshold which are responsible for the large rates of production of the singlet-P states [6]-[8]. The presence of these intermediate states in the transitions $\Upsilon(5 \mathrm{~S}) \rightarrow \pi^{+} \pi^{-} h_{b}(\mathrm{nP})$ and $\Upsilon(6 \mathrm{~S}) \rightarrow \pi^{+} \pi^{-} h_{b}(\mathrm{nP})$ enables the vector-to-pseudoscalar transitions without suppression which would otherwise occur due to the necessary $b$-quark spin flip in these transitions.

The $Z_{b}(10610)$ and $Z_{b}(10650)$ have masses very close to the thresholds for $B \bar{B}^{*}$ and $B^{*} \bar{B}^{*}$, respectively, which indicates possible molecular structure for these states, or at the very least a very substantial molecular composition in the $Z_{b}$ wave functions. The fact that these states have been observed principally to decay to these $B$-meson pair combinations strengthens this conclusion [8]. Furthermore, spin-parity measurement, total widths, and production rates are also consistent with expectations for molecular states. The only possible contraindication for molecular identity is the fact that the $Z_{b}$ states lie slightly above the mass thresholds (though not significantly). Molecular states' masses should be below threshold - although it must be 

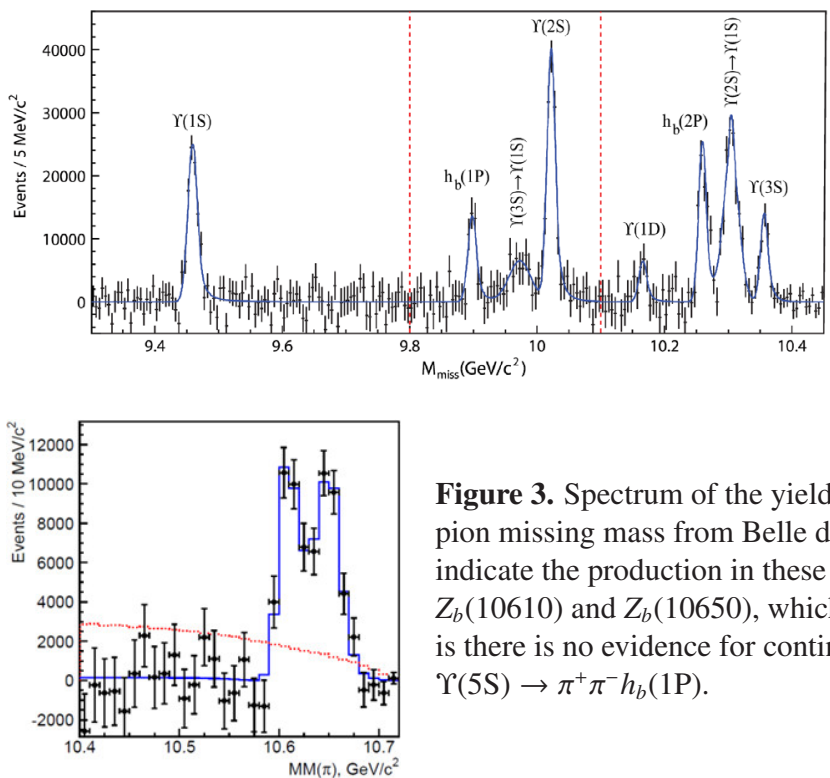

Figure 2. Dipion missing mass spectrum from Belle, for data taken at $\Upsilon(5 S)$ [5]. Indicated in this spectrum are transitions both to vector $\Upsilon(\mathrm{nS})$ states, singlet $h_{b}(\mathrm{nP})$ states, and reflections of dipion transitions among lower $\Upsilon(\mathrm{nS})$ states.

Table 2. Branching fractions for observed products of $Z_{b}(10610)$ and $Z_{b}(10650)$ decays [8]. Branching fractions reported assume that these transitions saturate the total decay rates of the two states.

\begin{tabular}{lll}
\hline Channel & \multicolumn{2}{c}{ Branching Fraction (\%) } \\
& $Z_{b}(10610)$ & $Z_{b}(10650)$ \\
\hline$\Upsilon(1 \mathrm{~S}) \pi^{+}$ & $0.54_{-0.13}^{+0.16}+0.11$ & $0.17_{-0.06}^{+0.07+0.03}$ \\
$\Upsilon(2 \mathrm{~S}) \pi^{+}$ & $3.61_{-0.59}^{+0.76}+0.79$ & $1.39_{-0.38}^{+0.48}+0.34$ \\
$\Upsilon(3 \mathrm{~S}) \pi^{+}$ & $2.15_{-0.45}^{+0.55}+0.60$ & $1.63_{-0.53}^{+0.53}+0.39$ \\
$h_{b}(1 \mathrm{P}) \pi^{+}$ & $3.45_{-0.77}^{+0.87}+0.86$ & $8.41_{-2.12}^{+2.43}+1.49$ \\
$h_{b}(2 \mathrm{P}) \pi^{+}$ & $4.67_{-1.00}^{+1.24}+1.18$ & $14.7_{-2.8}^{+3.2}+2.8$ \\
$B^{+} \bar{B}^{* 0}+\bar{B}^{0} B^{*+}$ & $85.6_{-2.0}^{+1.5}+1.5$ & - \\
$B^{*+} \bar{B}^{* 0}$ & - & $73.7_{-4.4}^{+3.4}+2.7$ \\
\hline
\end{tabular}

acknowledged that masses of states may shift significantly due to coupled channel effects. Further study of these states is both warranted and eagerly anticipated in Belle II.

While we have learned a great deal about these states in our studies, there is much more to be learned - in particular, we would like to study the $Z_{b}$ production fractions as a function of energy across the $\Upsilon(5 S)$ and $\Upsilon(6 S)$ region. Also, while we have some understanding of the principal decay modes of the $Z_{b}$ states (see Table 2), a healthy $1-2 \mathrm{ab}^{-1}$ sample of $\Upsilon(5 S)$ expected for Belle II would give the opportunity both to determine whether other channels have significant contribution to the $Z_{b}$ total widths, and to determine more precisely the various decay branching fractions and angular distributions.

\section{Studies of $h_{b}(\mathrm{nP})$ and $\eta_{b}(\mathrm{nS})$ States}

The $h_{b}(\mathrm{nP})$ states were discovered as noted previously in $\pi^{+} \pi^{-}$transitions from $\Upsilon(5 \mathrm{~S})$, in a sample of $121.4 \mathrm{fb}^{-1}$. Subsequently transitions $\Upsilon(5 \mathrm{~S}) \rightarrow \eta h_{b}(1 \mathrm{P})$ were also observed and therefore a large sample of $\Upsilon(5 S)$, of order ten times the previous size, is something we'd like to accumulate to study this process. As shown in Figure 4, Belle made the observation of 

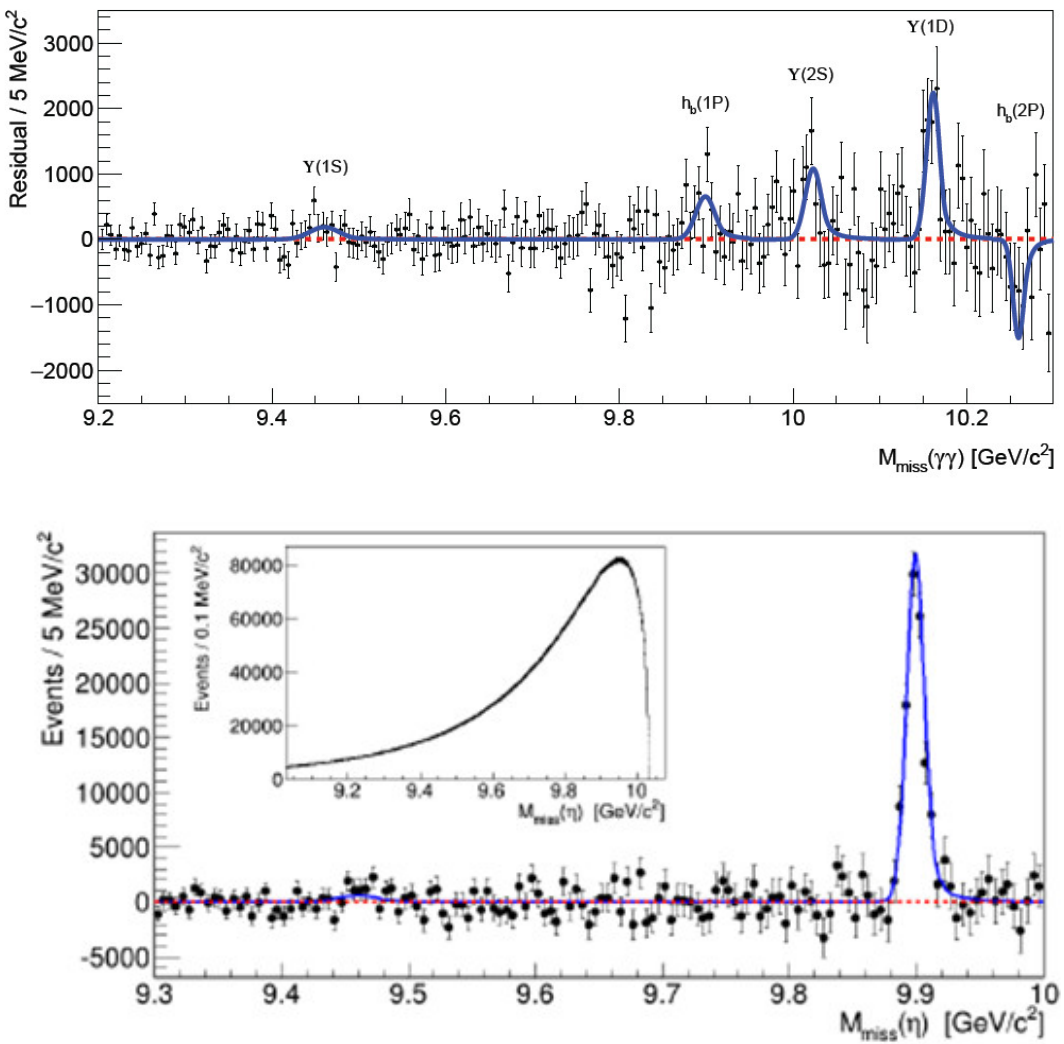

Figure 4. (top) Evidence for $\eta$ transitions from $\Upsilon(5 S)$ seen in $\eta$ missing mass from the Belle sample of $121.4 \mathrm{fb}^{-1}$. Peaks are observed for transitions to $\Upsilon(1 \mathrm{~S}), h_{b}(1 \mathrm{P}), \Upsilon(2 \mathrm{~S})$, and $\Upsilon(1 \mathrm{D})$. (bottom) Observation of $\Upsilon(4 \mathrm{~S}) \rightarrow \eta h_{b}(1 \mathrm{P})$ in the $\eta$ missing mass spectrum.

a very large branching fraction for $\Upsilon(4 \mathrm{~S}) \rightarrow \eta h_{b}(1 \mathrm{P})$, and this offers yet another avenue for accumulation of large $h_{b}(1 \mathrm{P})$ data sample. Naturally, then, there is a reasonable expectation that $\eta$ transitions from $\Upsilon(6 \mathrm{~S})$ may also be large enough to provide a useful sample of both $h_{b}(1 \mathrm{P})$ and $h_{b}(2 \mathrm{P})$.

As a result, data samples taken in Belle II at all three of the $\Upsilon(4 S), \Upsilon(5 S)$, and $\Upsilon(6 S)$ states have the potential to serve as a source of $h_{b}(\mathrm{nP})$, and with such samples, we can study both the decays of $h_{b}(\mathrm{nP})$ and those of $\eta_{b}(\mathrm{nS})$ by tagging $h_{b}(\mathrm{nP})$ in $\pi^{+} \pi^{-}$or $\eta$ transitions, and subsequently $\eta_{b}(\mathrm{nS})$ in hard radiative decays of higher $h_{b}(\mathrm{nP})$ which decay prolifically to the $\eta_{b}(\mathrm{nS})$. The branching fractions of $\Upsilon(4 \mathrm{~S})$ and $\Upsilon(5 \mathrm{~S})$ to produce $h_{b}(\mathrm{nP})$ are given in Table 3, and using them, and efficiencies from Belle II Monte Carlo study, we can expect usable samples (assuming $50 \mathrm{ab}^{-1}$ at $\Upsilon(4 \mathrm{~S})$ and $2 \mathrm{ab}^{-1}$ at $\Upsilon(5 \mathrm{~S})$ ) of millions of $h_{b}(\mathrm{nP})$ and $\eta_{b}(1 \mathrm{~S})$, and a few hundred thousand $\eta_{b}(2 \mathrm{~S})$. With such samples, we will have the opportunity to make precise measurements of both the lineshape for the M1 photons which are produced in the transitions, the $\eta_{b}(\mathrm{nS})$ masses and widths, and potentially some important decay branching fractions (e.g. $\eta_{b}(1 \mathrm{~S}) \rightarrow \gamma \gamma$, which is of fundamental importance as it is related directly to the $b \bar{b}$ wave function at the origin, and $\left.\eta_{b}(2 S) \rightarrow \pi^{+} \pi^{-} \eta_{b}(1 \mathrm{~S})\right)$.

It is likely that in Belle II, additionally, that a sample of order $300 \mathrm{fb}^{-1}$ will be taken at $\Upsilon(3 \mathrm{~S})$. As such, we can expect with such a sample to have another $600 \mathrm{k} \eta_{b}(1 \mathrm{~S})$ for study 
Table 3. Known branching fractions and expected yields in Belle II for the production of the singlet states $h_{b}(\mathrm{nP})$ and $\eta_{b}(\mathrm{nS})$ in decays of $\Upsilon(4 \mathrm{~S})$ and $\Upsilon(5 \mathrm{~S})$, assuming $50 \mathrm{ab}^{-1}$ at $\Upsilon(4 \mathrm{~S})$ and $2 \mathrm{ab}^{-1}$ at $\Upsilon(5 \mathrm{~S})$.

\begin{tabular}{lll}
\hline Process & Branching Fraction $(\%)$ & expected observed yield $\left(\times 10^{6}\right)$ \\
\hline$\Upsilon(4 \mathrm{~S}) \rightarrow \eta h_{b}(1 \mathrm{P})$ & $0.183 \pm 0.023$ & 4 \\
$\Upsilon(5 \mathrm{~S}) \rightarrow \pi^{+} \pi^{-} h_{b}(1 \mathrm{P})$ & $0.35 \pm 0.11$ & 8 \\
$\Upsilon(5 \mathrm{~S}) \rightarrow \pi^{+} \pi^{-} h_{b}(2 \mathrm{P})$ & $0.60 \pm 0.20)$ & 1.4 \\
$h_{b}(1 \mathrm{P}) \rightarrow \gamma \eta_{b}(1 \mathrm{~S})$ & $49.2 \pm 5.7_{-3.3}^{+5.6}$ & 3.7 \\
$h_{b}(2 \mathrm{P}) \rightarrow \gamma \eta_{b}(1 \mathrm{~S})$ & $22.3 \pm 3.8_{-3.1}^{+3.3}$ & 0.3 \\
$h_{b}(2 \mathrm{P}) \rightarrow \gamma \eta_{b}(2 \mathrm{~S})$ & $47.5 \pm 10.5_{-7.7}^{+6.8}$ & 0.6 \\
\hline
\end{tabular}

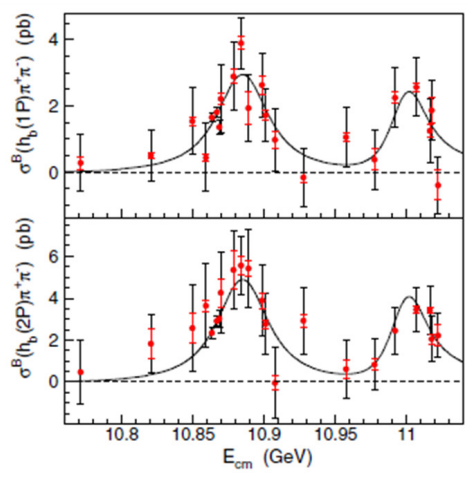

Figure 5. $h_{b}(\mathrm{nP})$ cross section as a function of center of mass energy, showing the resonance curves of both $\Upsilon(5 S)$ and $\Upsilon(6 S)$.

of $\eta_{b}(1 \mathrm{~S})$. One of the more important reasons to look particularly at this sample is the difference in mass measurements for $\eta_{b}(1 \mathrm{~S})$ that persists between the analyses of M1 transitions $\Upsilon(\mathrm{nS}) \rightarrow \gamma \eta_{b}(1 \mathrm{~S})$ by BaBar [9] and CLEO [10], and the analysis of E1 transitions $h_{b}(\mathrm{nP}) \rightarrow \gamma \eta_{b}(1 \mathrm{~S})$ by Belle [11].

\section{Studies of Transitions from the $\Upsilon(6 S)$}

While the $h_{b}(\mathrm{nP})$ states can be studied in significant detail in large data samples Belle II expects to accumulate at $\Upsilon(4 S)$ and $\Upsilon(5 S)$, it is also likely that we can add to our knowledge of these states (and possibly the 3S singlet states) by studying transitions from $\Upsilon(6 S)$ as well. In Belle's small data sample of a $5.6 \mathrm{fb}^{-1}$ in the vicinity of $\Upsilon(6 \mathrm{~S})$, transitions to both $\pi^{+} \pi^{-} \Upsilon(1 \mathrm{~S})$ and $\pi^{+} \pi^{-} h_{b}(\mathrm{nP})$ were observed [12]. The yield of $h_{b}(\mathrm{nP})$ states is shown in Fig. 5. While production of both $h_{b}(\mathrm{nP})$ states had been previously established in data taken at the $\Upsilon(5 \mathrm{~S})$ resonance [5], this study revealed $3.5 \sigma$ and $5.3 \sigma$ signals for $h_{b}(1 \mathrm{P})$ and $h_{b}(2 \mathrm{P})$, respectively at the $\Upsilon(6 \mathrm{~S})$. Furthermore, the $\Upsilon(5 \mathrm{~S})$ also has been observed to decay to $\eta h_{b}(1 \mathrm{P})$, so a search for similar transitions to both $h_{b}(\mathrm{nP})$ states would prove interesting. For these reasons, then, any appreciable data sample taken at $\Upsilon(6 \mathrm{~S})$, can serve as an additional and revealing source of $h_{b}(\mathrm{nP})$ decays.

What is of more interest, however, is the resonant substructure in these transitions from $\Upsilon(6 S)$ as compared to transitions from $\Upsilon(5 S)$. In Belle, the resonant substructure of these transitions was investigated, and, as shown in Fig. 6, one very interesting fact stands out: while transitions through at least one of the charged $Z_{b}$ states can be established, it cannot be definitively stated whether such transitions take place through both (rather than just one) state. 

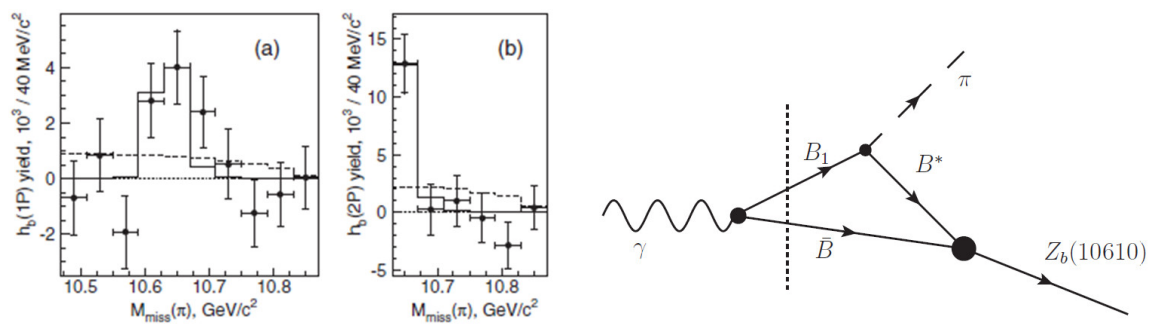

Figure 6. (left) Evidence for $Z_{b}$ produced as intermediate states in $\Upsilon(6 \mathrm{~S}) \rightarrow \pi^{+} \pi^{-} h_{b}(\mathrm{nP})$ transitions. The statistics are insufficient to determine the relative population of the two $Z_{b}$ states in these decays. (right) Potential decay diagram for $\Upsilon(6 \mathrm{~S})$ if its wave function has a sizeable $B_{1} B$ component, as suggested in Ref. [14].

The $\Upsilon(6 S)$, like the other vector states above open bottom threshold, is almost certainly not a simple $b \bar{b}$ bound state; at the very least, coupled channel considerations play a large role in its decays. Along these lines, a very interesting comparison that has been suggested is between the $\Upsilon(6 \mathrm{~S})$ and the $Y(4260)$. Both these states transition to lower narrow heavy quarkonium states by dipion transitions, and do so through charged quarkonium-like states. The mass of $\Upsilon(6 \mathrm{~S})$ is very near the threshold for the production of a $B_{1} B$ pair, just as the $Y(4260)$ is near the threshold for the charm analog, $D_{1} D$. Because of its unusual properties, and the proximity to the $D_{1} D$ threshold, the $Y(4260)$ has been interpreted as a possible molecule [13] - and the $\Upsilon(6 S)$ might likewise be interpreted. One important indicator for the possibility of $\Upsilon(6 \mathrm{~S})$ as a $B_{1} B$ molecule would be the exclusive transition through the lower $Z_{b}$ state, $Z_{b}(10610)$ [14] via the diagram in Fig.6. As already noted, the current small data sample near $\Upsilon(6 S)$ is insufficient to rule out or require $Z_{b}(10610)$ dominance in its transitions to lower bottomonia. More data then are needed to settle this very interesting question.

A data sample of $\sim 20 \mathrm{fb}^{-1}$ at the peak of $\Upsilon(6 \mathrm{~S})$ would enable more detailed studies of the $\Upsilon(6 S) \rightarrow \pi^{ \pm} Z_{b}{ }^{\mp}$ process and determine the question of relative production of the $Z_{b}(10650)$ and $Z_{b}(10610)$. Such a sample at $\Upsilon(6 S)$ would also make possible searches for expected $Z_{b}$ partners [15] in the decays $\Upsilon(6 S) \rightarrow \gamma W_{b}$ and possibly $\Upsilon(6 S) \rightarrow \pi^{+} \pi^{-} W_{b}$. These transitions would allow searches for transitions to lower narrow bottomonia by analogy to our observations of states in $\Upsilon(5 \mathrm{~S})$ decays, including possibly some of the as-yet observed narrow states $\Upsilon(2 D), h_{b}(1 \mathrm{P})(3 P)$, and $\Upsilon(1 F)$. Further, a fine grained search for $\pi^{+} \pi^{-} \Upsilon(\mathrm{nS})$ with high statistics (say, $1-2 \mathrm{fb}^{-1}$ per data point) in the region above $10.7 \mathrm{GeV}$ could potentially add insight as the thresholds for production of the $Z_{b}$ and $W_{b}$ states are crossed.

\section{Studies of the $\Upsilon(1 D, 2 D)$ States}

Another of the narrow sub-threshold states of bottomonium that can be studied with Belle II data is the $\Upsilon(1 \mathrm{D})$ and $\Upsilon(2 D)$ states - knowledge of these $\mathrm{L}=2 \mathrm{D}$-wave states of bottomonium is relatively limited. The triplet of $1 \mathrm{D}$ states (having total angular momentum $J=1,2$, and 3 has thus far only been observed as an undifferentiated conglomerate in experimental papers from CLEO [16] in four-photon cascades in data taken at $\Upsilon(3 S)$ and BaBar [17] in their $\Upsilon(3 \mathrm{~S})$ data sample by reconstruction of the $\Upsilon(1 \mathrm{D}) \rightarrow \pi^{+} \pi^{-} \Upsilon(1 \mathrm{~S}) \rightarrow \pi^{+} \pi^{-} \ell^{+} \ell^{-}$final state (See Fig. 7). The $\Upsilon(2 D)$ levels are completely unknown at present.

In data taken at Belle, we have seen an indication (though not statistically significant) of the process $\Upsilon(5 \mathrm{~S}) \rightarrow \pi^{+} \pi^{-} \Upsilon(1 \mathrm{D})$ in our $h_{b}(\mathrm{nP})$ discovery paper [5], and most recently we have discovered the transition $\Upsilon(5 S) \rightarrow \eta \Upsilon(1 \mathrm{D})$ (See Fig. 4) but again in these cases, we only 

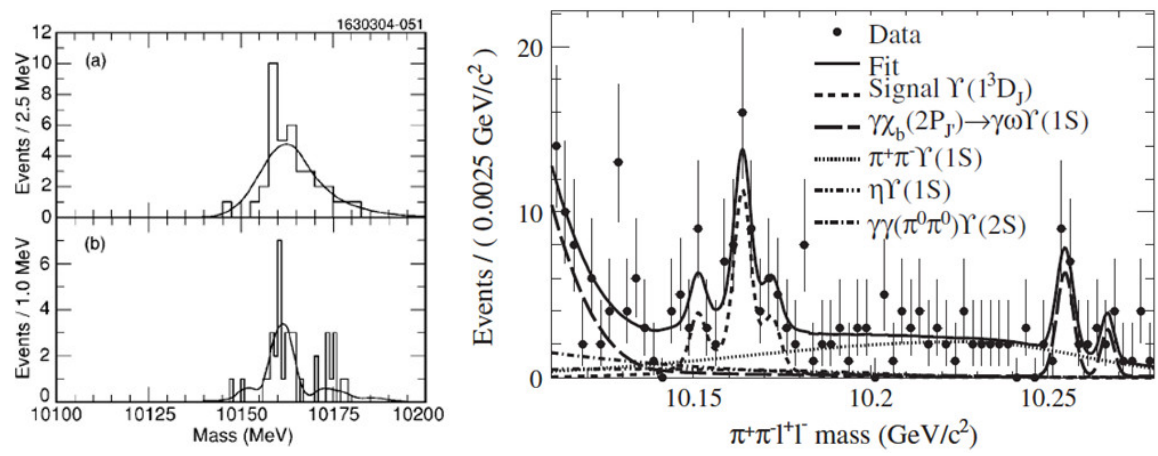

Figure 7. Observation of the $\Upsilon(1 \mathrm{D})$ states by (left) CLEO [16] and (right) BaBar [17].

have a broad observation of a state or states at the expected $\Upsilon(1 \mathrm{D})$ mass. For the purposes of understanding these high angular momentum states, it will be essential that we have the ability to differentiate the members of the triplet.

We also would like to observe decays of the $\Upsilon(1 \mathrm{D})$ states in addition to the radiative transitions to lower $\chi_{b J}(n P)$ states that have already been observed. Examples of the most likely transitions include $\Upsilon(1 \mathrm{D}) \rightarrow \pi^{+} \pi^{-} \Upsilon(1 \mathrm{~S})$ or $\eta \Upsilon(1 \mathrm{~S})$. The much larger data samples of Belle II should allow us a good opportunity both to isolate one or more of the $\Upsilon(1 \mathrm{D})$ states from the others and possibly to observe the $\Upsilon(2 D)$ triplet expected to lie near $10.45 \mathrm{GeV} / \mathrm{c}^{2}$.

The BaBar observation [17] suggests the possibility of resolution of the three states with an $\Upsilon(3 \mathrm{~S})$ data sample approaching $250-300 \mathrm{fb}^{-1}$, which we expect to collect sometime early in Phase III of Belle II operations. Furthermore the $J^{P C}=1^{--}$member of the $\Upsilon(1 \mathrm{D})$ and $\Upsilon(2 D)$ triplets could also be reached directly by scanning the $e^{+} e^{-}$beams across each resonance. The beauty of this potential search is that by it, both the mass and possibly the full width of the $1^{--}$members of the triplets can be established. With predicted $\Gamma_{e e}$ partial widths of order $1 e V$, a $2 \mathrm{fb}^{-1}$ per point scan would result in a signal of greater than $5 \sigma$ significance.

\section{Conclusions}

In addition to its ambitious plans in $\mathrm{CP}$ violation and searches for extremely rare decays of B mesons which will shed light on new physics, the Belle II experiment has the opportunity and indeed excellent prospects to shed light on the nature of strongly interacting systems such as conventional states of bottomonium and the exotic bottomonium-like states recently discovered.

\section{Acknowledgments}

The author thanks the organizers of the 6th International Conference on Exotic Atoms and Related Topics for the invitation to speak, his Belle Collaboration colleagues for their various contributions of analyses presented, and the National Science Foundation for their support of this work through Grant \#1506412.

\section{References}

[1] Y. Ohnishi et al., “Accelerator design at SuperKEKB,” PTEP 2013, 03 A011 (2013) 
[2] M. Baszczyk et al. (SuperB Collaboration), INFN-13-01-PI, LAL-13-01, SLAC-R-1003, arXiv:1306.5655.

[3] T. Abe et al. (Belle Collab.) 2, KEK-REPORT-2010-1, arXiv:1011.0352.

[4] M. Nayak et al. , PoS BEAUTY 2016059 (2016).

[5] A. Bondar et al. (Belle Collab.) ,Phys. Rev. Lett. 108, 122001 (2001).

[6] I. Adachi et al. (Belle Collab.), Phys. Rev. Lett. 108, 032001 (2001).

[7] P. Krokovny et al. (Belle Collab.), Phys. Rev. D 88, 052016 (2013).

[8] A. Garmash et al. (Belle Collab.), Phys. Rev. Lett. 116, 212001 (2016).

[9] B. Aubert etal. (BaBar Collab.), Phys. Rev. Lett. 101, 071801 (2008); Phys. Rev. Lett. 103, 161801 (2009).

[10] G. Bonvicini et al. (CLEO Collab.), Phys. Rev. D 81, 031104(R) (2010).

[11] R. Mizuk et al. (Belle Collab.) Phys. Rev. Lett. 109, 232002 (2012).

[12] R. Mizuk et al. (Belle Collab.) Phys. Rev. Lett. 117, 142001 (2016).

[13] Q. Wang, C. Hanhart, and Q. Zhao, Phys. Rev. Lett. 111, 132003 (2013).

[14] M. Voloshin, B2TiP Pittsburgh 2016.

[15] A. E. Bondar et al. Phys. Rev. D 84, 054010 (2011).

[16] G. Bonvicini et al. (CLEO Collab.), Phys. Rev. D 70, 032001 (2004).

[17] P. del Amo Sanchez et al. (BaBar Collab.), Phys. Rev. D 82, 11102 (2010). 